\title{
Restoring for Natural Authenticity
}

In the practice of restoration we strive to restore healthy ecosystems, which I characterize as those that meet the following criteria: A restored healthy ecosystem contains a broad representation of species from the kind of ecosystem that is being restored. These consist of indigenous species to the greatest practicable extent and represent all of the structural and functional species-groups necessary for the development of that ecosystem. The physical environment has been suitably prepared to sustain these species as reproducing populations. Insofar as possible, potential threats to the restored ecosystem from the surrounding area have been eliminated. The restored ecosystem is apparently functioning reasonably well in terms of energy flow and nutrient cycling. The restored ecosystem is dynamic with respect to species recruitment, population growth, food-web development, accumulation of biomass, carbon storage, moisture balance, microclimatic control, interspecific interactions, and the differentiation of habitat for specialized species. The restored ecosystem is sufficiently resilient to endure normal stress events in the local environment, such as periodic fire or seasonal flooding. It is self-sustaining, although it may and probably will need periodic post-restoration management to resist pervasive human influences, such as those caused by damaging land-use activities and the inexorable spread of exotic species. The restored ecosystem has the potential to persist indefinitely, although it may change in appearance in response to ecosystem dynamics and stress. It is also potentially capable of evolving in response to long-term environmental changes in geomorphology, climate, sea level, etc. In addition to these criteria, the restored ecosystem provides natural services of economic, cultural, and aesthetic consequence, and it harbors germ plasm, can accommodate rare and endangered species, and otherwise conserves biodiversity.

In this article I explore two questions of authenticity that are raised by the concept of restoration. First: Is what we restore natural? In other words, does a restored ecosystem exhibit natural authenticity? By this I mean an ecosystem that developed in response to natural processes and that lacks indications of being intentionally planned or cultured. Or should every restored ecosystem be considered artifice and merely a replica of nature? The second question is: To what degree can we return authenticity in a restored ecosystem relative to the original one it replaces? In other words, can we really put the ecosystem back the way it was with historical authenticity?

The answer to the first question is an emphatic yes. The principal task of restoration practice is to initiate or accelerate ecosystem dynamics - that is, natural autogenic processes. Once these processes of self-renewal have fully resumed, the practitioner's work is finished. Thereafter, the ecosystem continues to restore itself. Self-renewal can generate numerous potential endpoints, owing to the complexity and unpredictability of ecosystem dynamics. An endpoint in this context is the expression of a restored ecosystem at a particular time or at a given stage in development. The practitioner can exert limited control of the endpoint by prescribing the initial floristic composition and by controlling development until a newly restored ecosystem is capable of independent self-renewal. However, a specified endpoint is not assured. Even if the specified endpoint is attained, the restored ecosystem is subject to continued development and evolution. For these reasons a restored ecosystem is not artifice. Instead, it selforganizes through natural processes and is naturally authentic.

Restoration would be artifice if it were a form of farming, gardening, intensive forestry, active landscaping, or bio-engineering. The reason is that land that is cultivated, landscaped, or engineered is subject to manipulation in all stages of its development. Such manipulation assures the attainment of a predetermined endpoint or of arrested development at a desirable stage. The goal of farming is not to initiate natural processes but to produce commodities. The goal of landscaping is to fashion a particular landscape for a specified purpose. The goal of bio-engineering is to provide a service such as erosion control or nutrient uptake. Restored ecosystems also provide goods and services, and one rationale for conducting restoration is to realize an array of sustainable benefits at minimal cost. The difference is that these benefits accrue from natural systems without human assistance; they are not intentionally produced or engineered in managed systems.

Inow turn to the second question. Can we put an ecosystem back the way it was with historical authenticity? The answer is no for the very reason that a restored ecosystem is natural and not artifice: restoration cannot guarantee a particular endpoint. We might get lucky and achieve an endpoint that is historically authentic. Otherwise, we can only achieve historical authenticity by manipulating the ecosystem in its later stages of development. When we do this, the project becomes artifice, and we have sacrificed natural authenticity.

Attainment of historical authenticity requires documentation by means of a deliberate evaluation. A reference ecosystem must be selected to serve initially as a model for restoration and later as a basis for comparison to ascertain the degree of historical authenticity that was achieved. A range of potential reference sites is available. The reference could be the original, pre-disturbance ecosystem. It could be another ecosystem of the same kind that exists locally. It could also be a generalized ecosystem that was distilled from ecological inventories at multiple localities. The 
choice of the reference is critical, because the selection of one reference instead of another will affect what is meant by historical authenticity. The scales that are selected for inventorying the reference will also affect how historical authenticity is evaluated. For example, the description of a reference that was prepared from satellite imagery will have little resemblance to one that was derived from data in small quadrats.

To evaluate a restored ecosystem, the restorationist specifies particular attributes of the reference system, then sees whether they have been incorporated in the restored ecosystem. The selection of attributes and how they are weighted introduces judgment into the evaluation process. The list of attributes will likely be limited, because reference sites are not generally inventoried in detail, thereby injecting the bias of an incomplete comparison. The evaluation will likely occur before the restored ecosystem has attained the same level of ecological maturity as its reference. The evaluator must apply professional judgment to breach this gap in development. Judgment is required at each step in evaluation, and an evaluation is unavoidably subjective. We can still claim that we "put it back the way it was," but only with the candid admission that our assertion relies on the judgment of the evaluator. This reliance moves the evaluation of historical authenticity away from science and towards artistic criticism.

We can largely extract ourselves from this conundrum if we broaden what we mean by historically authentic to include any endpoint that is expressed, or that could reasonably be expressed, within the entire ecoregion for the kind of ecosystem being restored. This broadened conception of the reference enhances our ability to restore with historical authenticity and reduces the subjectivity in its evaluation. We are not restoring a site to its former manifestation of biodiversity. Instead, we are returning one of many possible authentic expressions of the regional ecosystem. By doing so, we acknowledge the reality of multiple endpoints in ecosystem development.

The concept of historical authenticity as a requisite for restoration harbors a bothersome inconsistency. If one were to create a healthy ecosystem that was entirely lacking in historical authenticity, it could not be designated as restoration. But if this same ecosystem were damaged, it could be restored with historic authenticity, because a reference would be readily available: the damaged ecosystem serving as the reference ecosystem for its own restoration. It seems incongruous to call the latter restoration but not the former.

Our professional identity as restorationists is obscured if we insist that ecological restoration has to reflect historical authenticity. Many practitioners restore healthy ecosystems with natural but not with historical authenticity. Either the physical environment was altered or the surrounding landscape was modified to the point that another kind of ecosystem had to be substituted for the historic ecosystem. Some environments have been so severely modified that only a novel ecosystem can be restored-that is, one with a truly original, invented species composition or community structure. The production of such substitute ecosystems has been called rehabilitation, which connotes at best a healthy ecosystem containing natural, but not historical, authenticity. Restoration and rehabilitation are points along a continuum that defies objective separation. Perhaps the majority of what has been called restoration during the past 25 years is better called rehabilitation. Articles published in professional journals commonly proffer rehabilitation projects indiscriminately as restoration. If we insist on historical authenticity in restoration, then at

\section{Can we put an ecosystem back the way it was with historical authenticity? The answer is no.}

least a large minority of practitioners, if not the majority, are rehabilitationists who have never engaged in restoration.

To summarize, historical authenticity is fluid, because its characterization varies with the reference selected and with the scale at which the reference is described. Once historical authenticity is characterized, it cannot be attained in restoration projects except by luck. If it is attained it cannot be evaluated objectively. Historical authenticity contains an internal inconsistency; it cannot be incorporated in restoration programs that require substitute ecosystems; it causes confusion with respect to the characterization of the restoration profession; and it is ignored by authors and editors who fail to distinguish historically authentic restoration from rehabilitation.

In spite of its metaphorical strength, the concept of historical authenticity is more of a burden than a benefit, and it should be retired. To do this, I offer an alternative for defining ecological restoration. We could say that we have not restored an ecosystem per se. Instead, we restored a specific geographic location by re-creating a healthy ecosystem where one had been degraded or destroyed. By doing so, we concede that it is more important for a given place to support a healthy ecosystem than it is to concern ourselves with the details of the historic system that could have been considered as its model. We are already restoring places with ecosystems, and perhaps it is time to start acknowledging what we really do.

The principal rationale for restoring ecosystems is not to revive the past in order to indulge our nostalgia, but to secure our future by restocking a dangerously depleted global inventory of natural areas. To accomplish that task, restoring for natural authenticity is paramount. Historical authenticity warrants continued recognition only as a coincidental amenity.

Andre F. Clewell

Andre F. Clewell owns A. F. Clewell, Inc., which specializes in ecological restoration and is located at 98 Wiregrass Lane, Quincy FL 32351, USA; 850/875-3868; FAX 850/875-1848; e-mail clewell@tds.net. 